\title{
Hydrophobization of the surface of mineral forms during cryogenic treatment of highly clayey sands
}

\author{
Grigoriy Shirman $^{1 *}$, Andrey Matveyev ${ }^{1}$ and Svetlana Salomatova ${ }^{1}$ \\ ${ }^{1}$ Chersky Mining Institute of the North, Siberian Branch, Russian Academy of Sciences, laboratory \\ “Concentration of Mineral Wealth”, 678980, Republic of Sakha (Yakutia), Yakutsk, Lenin str. 43.
}

\begin{abstract}
The results of experiments in a laboratory flotation device for assessing the effect of cryogenic treatment on the surface properties of natural highly dispersed mineral mixtures, such are high-clay sands, are presented. Experiments have shown that cryogenic treatment of sands significantly increases their hydrophobic properties, which is confirmed by an increase in the yield of the flotation foam product without using collecting reagents, and there is also an increase in the yield of the foam product depending on the number of freeze-thaw cycles. This phenomenon confirms the effect of increased disintegration of highly clayey sands in an air-water environment after cryogenic treatment. An X-ray phase analysis of samples of foam (hydrophobic) and chamber (hydrophilic) products of flotation studies was carried out on an X-ray diffractometer D8 DISCOVER. Analyzes have shown that the separation products contain the same mineral phases, slightly differing in quantity, judging by the intensity of the peaks.
\end{abstract}

Keywords: highly clayey sands, washing, cryogenic treatment, freezing, hydrophobicity, reagents, foam product.

\section{Introduction}

In the course of mineral processing, the main role of disintegration is the release of useful mineral components from the binder clay mass for their subsequent separation according to physical and physicochemical characteristics [1]. The efficiency of dispersion of sandy-clay mixtures depends not only on the mineral and granulometric composition and the disintegration method used, but also on such factors as the preliminary treatment of the washed material. One of the options for increasing the efficiency of disintegration in relation to highly clayey sands is their cryogenic treatment, which is quite real for the conditions of the North [2].

The results of earlier experimental studies on a laboratory model of a closed washing drum to assess the effect of cryogenic treatment on the dynamics of the formation and destruction of dense clay aggregates showed that cyclic alternating impacts on clay aggregates have a significant effect on the adhesive properties of the material during its washing, an increase in the number of cycles reduces the dispersion time and the intensity

* Corresponding author: shirman@inbox.ru 
of the mass gain of lumps, which excludes the formation of pellets in the process of washing, and reduces the loss of valuable components [3].

The authors' research is related to the study of changes in the adhesion properties of the products of cryogenic treatment of highly clayey sands, which can explain the nature and dynamics of dispersion (washing) of sands.

The aim of this work is to study the effect of cryogenic treatment of highly clayey sands on their surface properties.

The idea of the work is to assess the dynamics of changes in hydrophobicity depending on freeze-thaw cycles by the flotation method by the foam product yield without using collectors. To intensify the process of flotation separation of hydrophobic particles, only a foaming agent was used to create a three-phase medium.

\section{Methodology of Experimental Study}

The experiments were carried out using samples with an initial moisture content of $25 \%$, for which, according to the results of preliminary experimental studies, the most characteristic effect of cryogenic treatment on dispersibility in a water-air three-phase system was established.

The studies were carried out on a laboratory flotation machine 240 FL-A with a chamber volume of 1.5 liters (Fig. 1).

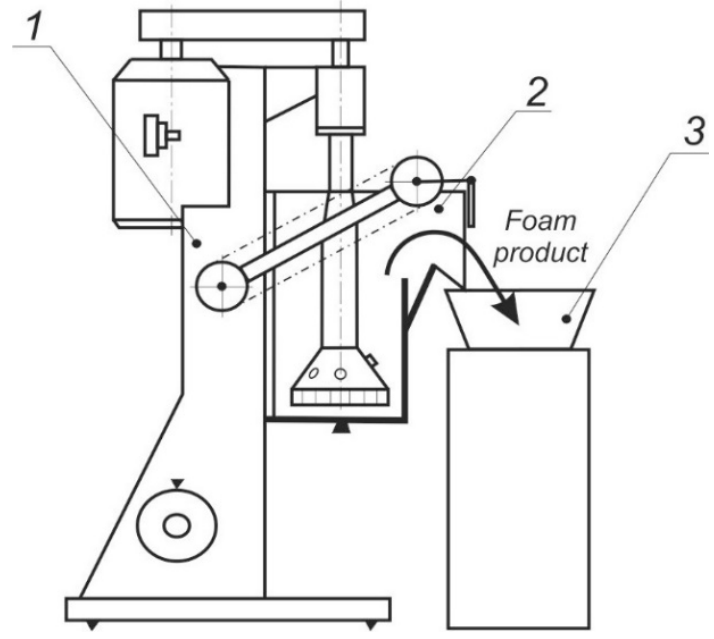

Fig. 1. Experimental stand.

1 - Flotation machine 240-FL-A; 2 - chamber flotation machine; 3 - container for the foam product.

As a starting material for the experiments, a fine-dispersed part of the initial highly clayey sands of the fraction $-0.2+0 \mathrm{~mm}$ (flotation size) was separated by sieving on laboratory sieves with a corresponding cell. Weighed portions of $210 \mathrm{~g}$, with a given moisture content of $25 \%$, were loaded into sealed containers and subjected to freezing in a refrigerator at a temperature of $253 \mathrm{~K}$ and thawing in a laboratory room at a temperature of $293 \mathrm{~K}$. The holding time for freezing and thawing was from 12 to 15 hours at each stage, the number of cycles was up to 2, samples that had not undergone cryogenic treatment were also studied.

The initial sample was loaded into the chamber of the flotation machine, the flotation process was carried out using a foaming agent (pine oil, $150 \mathrm{~g} / \mathrm{t}$ ) and without a collector reagent. The foaming agent feed was used to intensify the yield of hydrophobic particles. The foaming agent was supplied at relatively high concentrations to create a more abundant 
and stable foam, but eliminate mechanical carryover of hydrophilic particles. The flotation time for all experiments was 4 minutes, aqueous $\mathrm{pH}=6$. At the end of the experiment, the obtained foam and chamber products were dried, weighed, and then samples were taken for phase analysis on a D8 DISCOVER diffractometer. The work process is shown in Fig. 2.

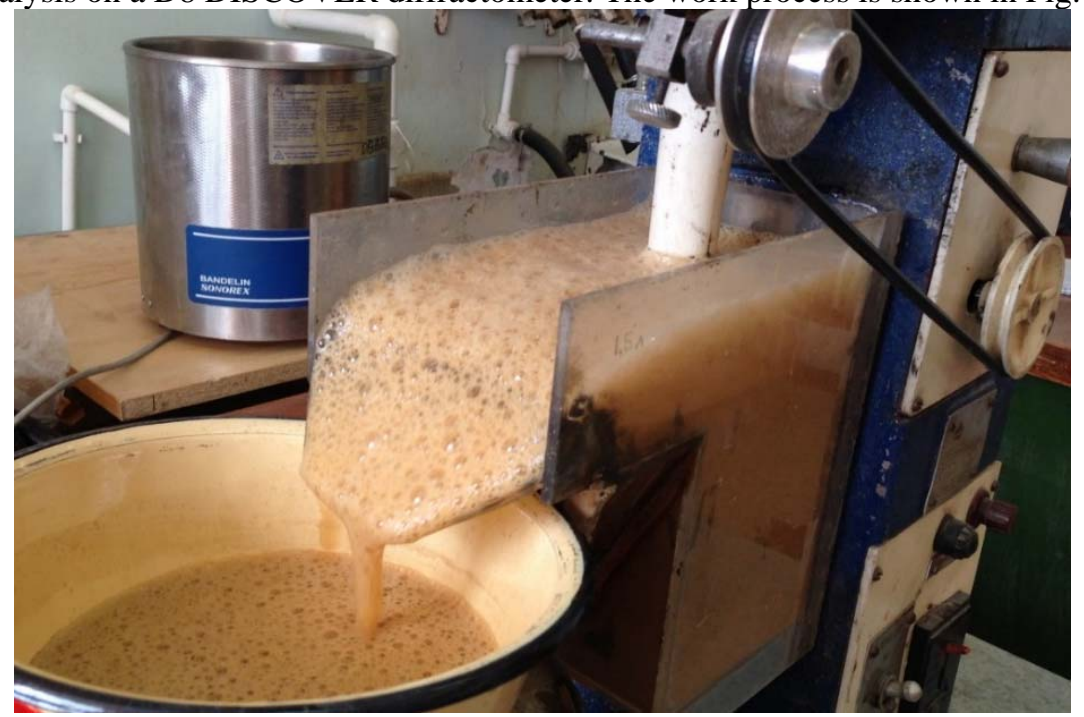

Fig. 2. The process of work on the FL 240-A flotation machine.

\section{Results}

Fig. 3 shows a summary diagram of the yield of the foam product for a floated sample depending on the number of freeze-thaw cycles with and without a reagent, as well as in comparison with samples that have not undergone cryogenic treatment.

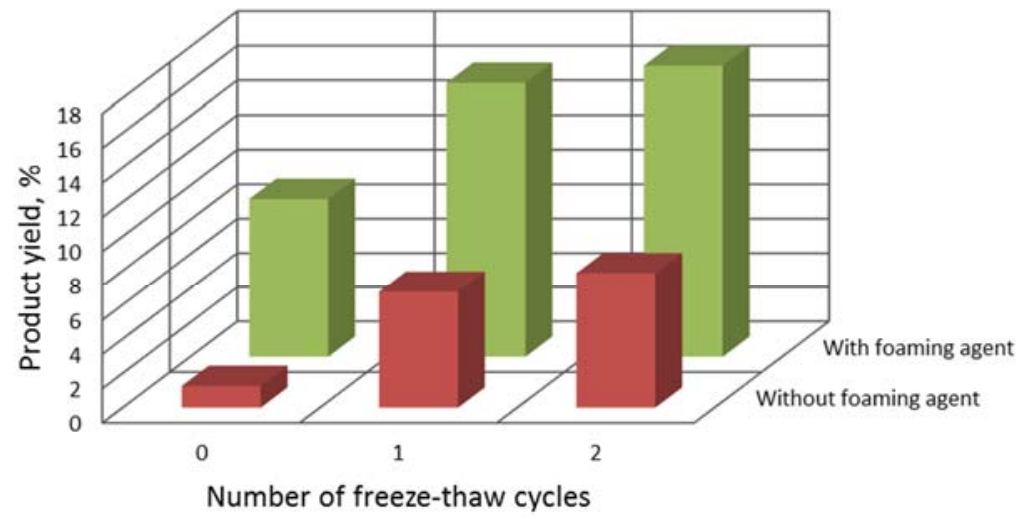

without foaming agent $\quad$ With foaming agent

Fig. 3. Yield of the foam flotation product with and without foaming agent, depending on the number of freeze-thaw cycles. 


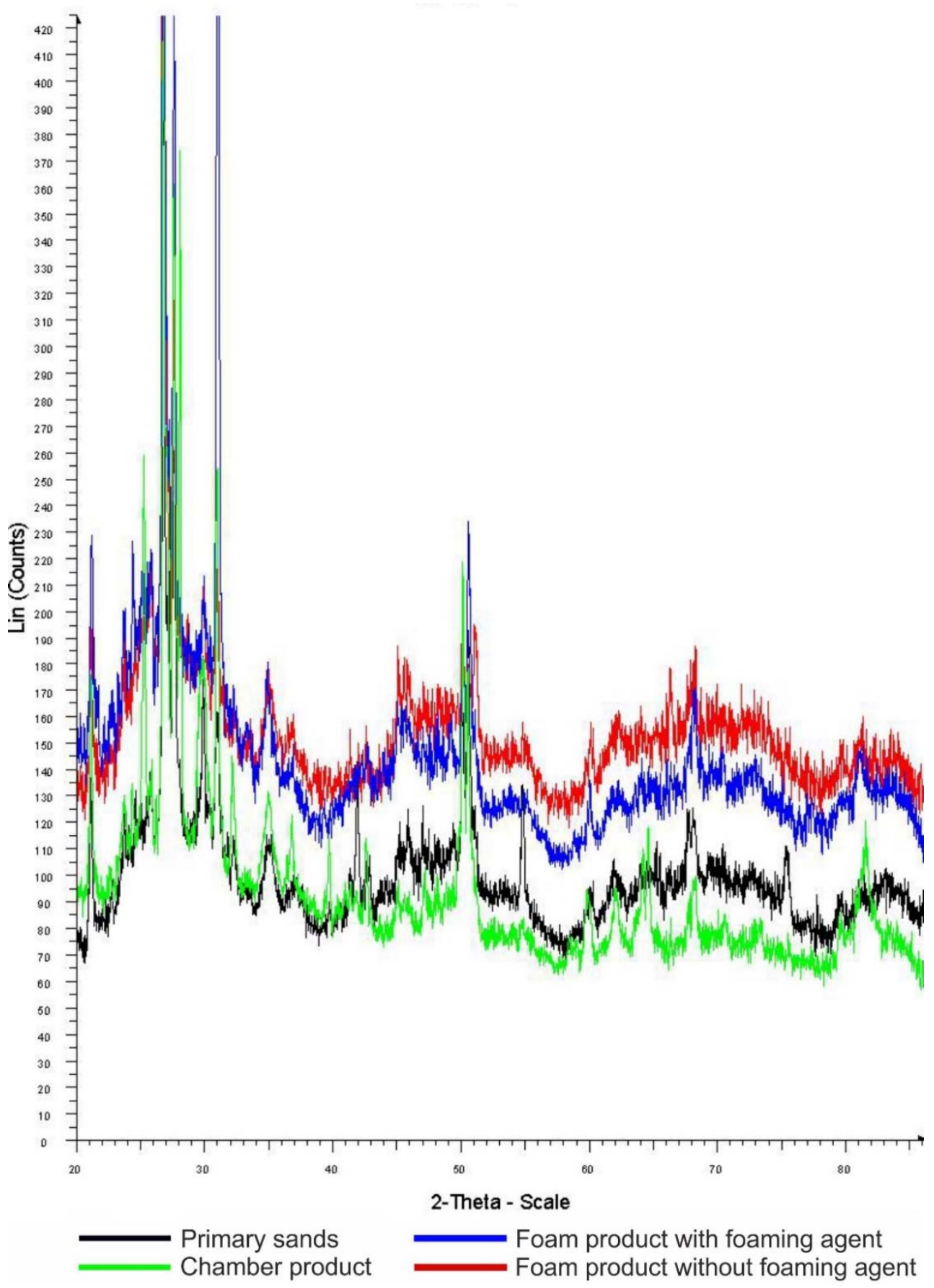

Fig. 4. General diffractogram of 4 samples.

As can be seen from the diagram, cryogenic treatment has a significant effect on the yield of foam materials (exhibiting hydrophobic properties) in all cases without exception. The use of a foaming agent significantly increases the yield of material with a foam product: for non-frozen samples from $1 \%$ to $10 \%$, and for cryogenically treated samples from $7 \%$ to $17 \%$. Preliminary studies indicate that for each sample, other things being equal, its characteristic value of the yield portion excludes the mechanical removal of 
material with foam, i.e. reflects the complete recovery of hydrophobic particles separated with the foam.

It is important that the thus acquired hydrophobicity of a finely dispersed material after thawing of the sample and subsequent freezing not only retains these properties, but also increases with additional freezing (cycle 2). It is quite obvious that this effect is associated with a change in surface properties (adhesion) during cryogenic treatment.

An X-ray phase analysis of samples of foam (hydrophobic) and chamber (hydrophilic) products of flotation studies, which was carried out on a D8 DISCOVER X-ray diffractometer with a GADDS (General Area Detector Diffraction System) made by Bruker (Germany), shows almost absolute identity of the phase composition of all separation products. Fig. 4 shows the general diffraction pattern of samples of flotation products (chamber and foam products). The diffraction pattern of the sample was obtained by moving the Vantec-500 detector at angles from 2-Theta $=8^{\circ}$ to 2-Theta $=88^{\circ}$ and recording the radiation energies for 300 seconds in each frame.

As can be seen from the diffractogram, the separation products contain the same mineral phases, slightly differing in quantity judging by the intensity of the peaks.

The quantitative analysis of the separation products and initial samples is carried out by Topas software (graphically based software for an analysis of the profile constructed by the least squares method) and is based on the dependence of the intensity of diffraction peaks on the phase content of the sample. Fig. 5 and 6 show a graph of the quantitative and mineral composition of the foam product obtained using the foaming agent. The composition of the chamber product is similar.

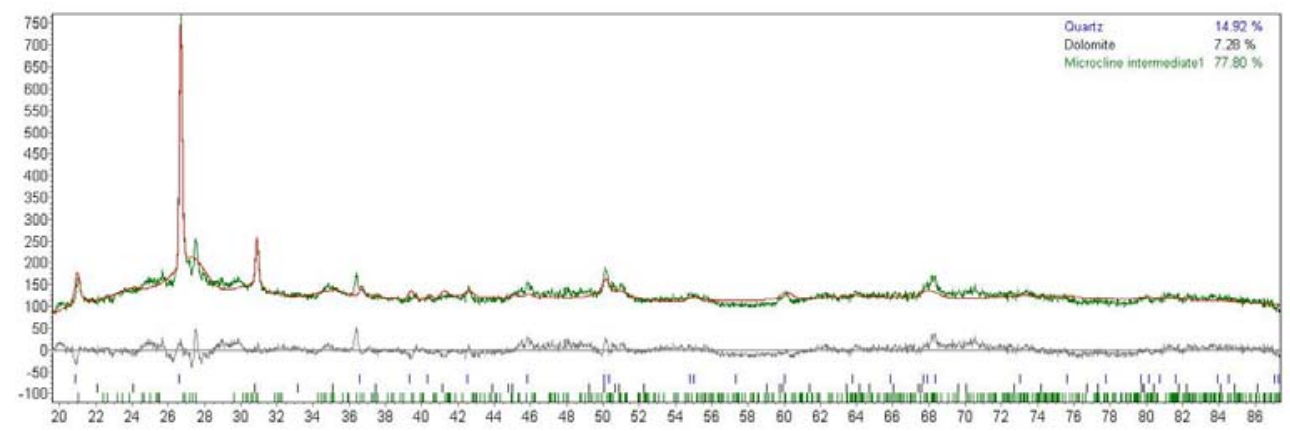

Fig. 5. Graph of the quantitative composition of the foam product obtained using a foaming agent.

Wt\% - Rietveld

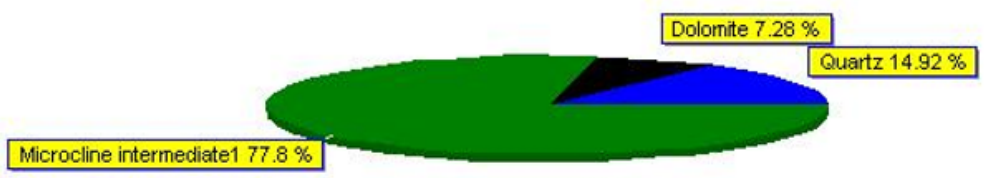

Fig. 6. Mineral composition of the foam product obtained using a foaming agent.

All samples contain the following minerals: quartz, microcline from the feldspar group and dolomite from the carbonate group. The approximate quantitative content of quartz in the samples is from $7 \%$ to $15 \%$, microcline - from $75 \%$ to $90 \%$, and dolomite - from $7 \%$ to $15 \%$. 


\section{Conclusion}

According to the results of the experiments, it was found that during cryogenic treatment, the surface hydrophobization occurs for all mineral forms. The phenomenon of hydrophobization of the mineral surface during cryogenic treatment largely explains the high disintegration effect of highly clayey sands in a water-air environment.

Research was carried out under the RFBR grant No. 18-45-140004 p_a

\section{References}

1. L.A. Vaisberg, L.P. Zarogatskiy, Physican and technical issues of mineral wealth development, 1, 99 (2003)

2. A.S. Kurilko, Experimental studies of the influence of freezing-thawing cycles on the physical and mechanical properties of rocks, (2004)

3. G.V. Shirman. IOP Conference Series: Earth and Environmental Science, 262, 012071 (2019) 